\title{
MOMENT MAPS ON SYMPLECTIC CONES
}

\author{
Suzana Falcão B. De Moraes and Carlos Tomei
}

We analyze some convexity properties of the image maps on symplectic cones, similar to the ones obtained by GuilleminSternberg and Atiyah for compact symplectic manifolds in the early 80's. We prove the image of the moment map associated to the symplectic action of an $n$-torus on a symplectic cone is a polytopic convex cone in $R^{n}$. Then, we generalize these results to symplectic manifolds obtained by special perturbations of the symplectic structure of a cone: we obtain sufficient (and essentially necessary) conditions for the image of the moment map associated to the perturbed form to remain unchanged.

Hamiltonian actions of tori and the images of their moment maps have been intensely studied in the eighties. According to the fundamental result, obtained independently by Atiyah [2] and Guillemin and Sternberg [4], the moment map of a Hamiltonian action of a torus on a compact symplectic manifold has for its image a convex polytope, spanned by the images of the fixed points of the action. More recently, Prato [10] proved a convexity result concerning the image of moment maps of torus actions on non-compact symplectic manifolds.

Theorem [10]. Let the torus $T^{r}$ act in a Hamiltonian fashion on the symplectic manifold $(X, \omega)$ and denote by $\Phi: X \rightarrow\left(\operatorname{Lie} T^{r}\right)^{*}=\mathbf{R}^{r}$ the corresponding moment map. Suppose that there exists a circle $S^{1}=\left\{e^{t} \xi_{0}\right\} \subseteq$ $T^{r}$ for some $\xi_{0} \in \operatorname{Lie} T^{r}$ such that $\Phi \xi_{0}=\left\langle\Phi, \xi_{0}\right\rangle$ is a proper function having a minimum as its unique critical value. Then $\Phi(X)$ is the convex hull of a finite number of rays in $\left(\operatorname{Lie} T^{r}\right)^{*}$.

In this paper, we prove a different kind of result, closer in spirit to perturbation theory: We start with a special non-compact symplectic manifold, described below, for which a similar convexity theorem holds, and consider the changes of the underlying symplectic structure which keep the image of the resulting moment maps unchanged.

Let $(X, \omega)$ be a symplectic cone with homothety group $\left\{\rho_{t}, t \in \mathbf{R}^{+}\right\}$so that $\rho_{t}^{*} \omega=t \cdot \omega$, for positive $t$, and compact base $X / \mathbf{R}^{+}$. Suppose that the torus $T^{r}$ acts symplectically on $(X, \omega)$ and that this action commutes with 
the $\mathbf{R}^{+}$-action. Since $(X, \omega)$ is a symplectic cone, the torus action is Hamiltonian (Proposition 1.1), for some associated moment map $\Phi:(X, \omega) \rightarrow \mathbf{R}^{r}$. Assume finally that there exists $\xi_{0} \in \mathbf{R}^{r}$ such that $\Phi_{0}=\left\langle\Phi, \xi_{0}\right\rangle>0$ (or, equivalently, that the image of $\Phi$ lies in an open half-space of $\mathbf{R}^{r}$ ). Then $X$ is diffeomorphic to $M \times \mathbf{R}^{+}$(again, from Proposition 1.1), where $M=\Phi_{0}{ }^{-1}(1)$ is a compact submanifold of $X$.

We prove the following convexity theorem in Section 1.

Theorem 1 (Convexity). The image of the moment map, $\Phi(X)$, is a polytopic convex cone.

We then consider in Section 2 symplectic forms $\Omega$ on $X$ which do not necessarily satisfy the homogeneity hypothesis, $\rho_{t}^{*} \Omega=t \cdot \Omega$, but for which the same $T^{r}$-action is still Hamiltonian, with moment map $\Psi$. We formulate hypothesis that are essentially necessary and sufficient for the ranges of the moment maps to coincide, i.e., so that $\Phi(X)=\Psi(X)$.

Theorem 2 (Rigidity). If $\lim _{t \rightarrow 0} \Psi(t, m)=0$ uniformly in $m \in M$ and $\lim _{t \rightarrow \infty} \Psi_{0}(t, m)=\infty$ (where $\left.\Psi_{0}=\left\langle\xi_{0}, \Psi\right\rangle\right)$ uniformly in $m \in M$, then the images of $\Phi$ and $\Psi$ coincide.

Notice that Theorem 2 only requires that the perturbed and unperturbed moment maps have similar behavior at the extremes of the cone. The essential necessity of these hypothesis will be shown by examples in the Appendix. The hypothesis at zero is equivalent to the integrability at zero of the oneform defined by the contraction of the perturbation $\Omega-\omega$ with the vector fields induced by the torus action. The perturbation of the symplectic form at infinity, on the other hand, is much more flexible.

An immediate consequence of the rigidity theorem is the result below, previously obtained by Guillemin (private communication) using $G$-invariant cohomology techniques.

Corollary. Let the torus $T^{r}$ act symplectically on a symplectic cone $(X, \omega)$, so that the torus action and the $\mathbf{R}^{+}$-action commute. Let $\Phi:(X, \omega) \rightarrow \mathbf{R}^{r}$ be the associated moment map. Assume that there exists a $\xi_{0} \in \mathbf{R}^{r}$ such that $\left\langle\Phi, \xi_{0}\right\rangle>0$. Let $\Omega$ be a symplectic form on $X$, such that the same $T^{r}$ - action is Hamiltonian with respect to it, with moment map $\Psi:(X, \Omega) \rightarrow \mathbf{R}^{r}$. If $\Omega-\omega$ is compactly supported, then the images of $\Phi$ and $\Psi$ coincide up to a translation.

Theorem 1 can be obtained using techniques similar to those in the proof of Prato's theorem, but we prefer to provide the self-contained argument in Section 1, which follows more closely the original proof of GuilleminSternberg in [4]. More precisely, by homogeneity, the image of the moment 
map $\Phi$ on $X$ is a cone over the image of its restriction to the compact manifold $M$. It is sufficient then to show the convexity of the image of the restriction, and this is accomplished by making use of the by now standard ingredients: The local form of the moment map and Morse theory for clean functions. The fact that $M$ is odd dimensional does not introduce any difficulty in this approach.

The proof of the rigidity theorem is by induction on the dimension of the cells associated to the usual stratification of the symplectic manifold by isotropy groups of the torus action. From the inductive hypothesis, the images of both unperturbed $(\Phi)$ and perturbed $(\Psi)$ moment maps, restricted to the closure of each cell up to a given dimension, agree (as sets, not pointwise). The key point in the induction step is to combine the fact that the images agree on the boundaries of the closures of the cells of higher dimension, with the fact that the images of the interiors of these cells 'leave no holes' in the convex span of their boundaries. The second fact follows from detailed study of the behavior of the moment map at interior faces (i.e., cells whose images are in the interior of the image of a larger cell). One of the nice features of the argument is that, without additional hypothesis, the perturbed moment map still omits the origin in its image.

In the Appendix we describe some examples and counter-examples. First, for the natural action of $T^{r}$ on $X=\mathbf{R}^{2 n}-\{0\}$ with the usual symplectic structure, we present all the symplectic structures for which the torus action remains Hamiltonian - from the answer, we will see that the hypothesis of the rigidity theorem are also necessary in this case. In the second example, we show how the behavior of the moment map at the origin can alter the image at infinity. The corollary above can be considered an additional example of both theorems applied to the more general symplectic cone.

Acknowledgements. The authors would like to thank Victor Guillemin for suggesting the study of moment maps on symplectic cones and Regina Souza, Nicolau Saldanha and Eugene Lerman for fruitful conversations. This work was partially supported by CNPq, SCT and FAPERJ, Brazil.

\section{A Convexity theorem for symplectic cones.}

Let $(X, \omega)$ be a symplectic cone with compact base. Explicitly, $X$ is a differentiable manifold with symplectic form $\omega$, admitting a homothety group $\left\{\rho_{t}, t \in \mathbf{R}^{+}\right\}$of diffeomorphisms such that $\rho_{t}^{*} \omega=t \cdot \omega$ and such that the base $X / \mathbf{R}^{+}$is a compact manifold. Suppose that the torus $T^{r}$ acts symplectically on $(X, \omega)$ and that its action commutes with the $\mathbf{R}^{+}$-action.

Given $\xi$ in the Lie algebra of $T^{r}=\operatorname{Lie} T^{r} \simeq \mathbf{R}^{r}$, let $\xi^{\sharp}$ be the symplectic vector field on $X$ associated to it. The conical structure of $(X, \omega)$ gives 
rise to a convenient description of the moment map of the torus action, as we will see in Proposition 1.1: Define $\Phi: X \rightarrow\left(\operatorname{Lie} T^{r}\right)^{*} \simeq \mathbf{R}^{r}$ by $\Phi^{\xi}=\langle\Phi, \xi\rangle=\iota\left(\xi^{\sharp}\right) \alpha$, for $\xi \in \operatorname{Lie} T^{r}$, where $\alpha=\iota(\Xi) \omega$, the vector $\Xi$ denotes the infinitesimal generator of the $\mathbf{R}^{+}$-action and $\iota(\eta) \beta$ is the contraction of the field $\eta$ with the form $\beta$. From now on, we assume that for some $\xi_{0} \in \operatorname{Lie} T^{r}, \Phi_{0}=\left\langle\Phi, \xi_{0}\right\rangle>0$ : In particular, $0 \notin \Phi(X)$.

Under these assumptions, we have the main result of this section.

Theorem 1 (Convexity). The image of the moment map, $\Phi(X)$, is a polytopic convex cone.

We make use of a number of simple properties of the moment map $\Phi$.

\section{Proposition 1.1.}

(1) The $T^{r}$-action on $(X, \omega)$ is Hamiltonian and $\Phi: X \rightarrow \mathbf{R}^{r}$ is indeed its moment map.

(2) $\Phi \circ \rho_{t}=t \cdot \Phi$, i.e., $\Phi$ is homogeneous.

(3) $X$ is diffeomorphic to the cartesian product $M \times \mathbf{R}^{+}$, where $M=$ $\Phi_{0}^{-1}(1)$ is a compact submanifold of $X$. In these coordinates, $\rho_{t}(m, s)=$ $(m, t s)$. In particular, the $\mathbf{R}^{+}$-action on $X$ is free.

(4) Let $\tau: G \times X \rightarrow X$ be an action of a connected compact group $G$ on $X$ that commutes with the $\mathbf{R}^{+}$-action. For each $x \in X$, let $G_{x}$ be the stabilizer group of $x$ and $[x]$ denote the corresponding element in $X / \mathbf{R}^{+}$. Then $G_{x}=G_{[x]}$ for $x \in X$.

(5) Only a finite number of subgroups of $T^{r}$ occur as stabilizer groups of points in $X$.

Proof. We first show that $d \Phi^{\xi}=-\iota\left(\xi^{\sharp}\right) \omega$. By the Weyl identity, $D_{\Xi} \omega=$ $d(\iota(\Xi) \omega)+\iota(\Xi) d \omega$. But $D_{\Xi} \omega=\omega$ and $\omega$ is closed, so $\omega=d(\iota(\Xi) \omega)=d \alpha$. Hence, if we define $\Phi^{\xi}=\iota\left(\xi^{\sharp}\right) \alpha, d \Phi^{\xi}=d\left(\iota\left(\xi^{\sharp}\right) \alpha\right)=D_{\xi^{\sharp}} \alpha-\iota\left(\xi^{\sharp}\right) d \alpha=$ $0-\iota\left(\xi^{\sharp}\right) \omega=-\iota\left(\xi^{\sharp}\right) \omega$. The homogeneity of the moment map is immediate. Now, consider the function $\Phi_{0}: X \rightarrow \mathbf{R}^{+}$and let $M=\Phi_{0}^{-1}(1) \subset X$. Since $\Phi_{0}=\iota\left(\xi_{0}^{\sharp}\right) \alpha,\left(\xi_{0}^{\sharp}\right)_{x} \neq 0$, for all $x \in X$. Now, $d \Phi_{0}=-\iota\left(\xi_{0}^{\sharp}\right) \omega$, so $\Phi_{0}$ has no critical values and $M$ is a manifold. Define $F: X \rightarrow M \times \mathbf{R}^{+}$to be $F(x)=\left(\rho_{1 / \Phi_{0}(x)}(x), \Phi_{0}(x)\right)$ with inverse $F^{-1}(m, t)=\rho_{t}(m)$. Both are smooth functions and so $X$ and $M \times \mathbf{R}^{+}$are diffeomorphic and $\mathbf{R}^{+}$acts freely on $X$. Clearly, $X / \mathbf{R}^{+} \simeq M$ and so $M$ is a compact manifold. The proof of (4) splits in two parts.

i) $G_{x} \subseteq G_{[x]}$.

Let $g \in G_{x}$, i.e., $\tau_{g}(x)=x$. Then $\tau_{g}([x])=\left[\tau_{g}(x)\right]=[x]$ and $g \in G_{[x]}$. 
ii) $G_{[x]} \subseteq G_{x}$.

Let $g \in G_{[x]}$, i.e., $\tau_{g}([x])=[x]$. In particular, $\tau_{g}(x)=\rho_{t}(x)$, for some $t \in \mathbf{R}^{+}$. We want to show that $t=1$. If we let $g$ vary in $G_{[x]}$, we get a map $A: G_{[x]} \rightarrow \mathbf{R}^{+}$where $A(g)=t$ defined by $\tau_{g}(x)=\rho_{t}(x)$. The map $A$ is a group homomorphism since if $A\left(g_{1}\right)=t_{1}$ and $A\left(g_{2}\right)=t_{2}$ then $\tau_{g_{1} g_{2}}(x)=$ $\tau_{g_{1}}\left(\tau_{g_{2}}(x)\right)=\tau_{g_{1}}\left(\rho_{t_{2}}(x)\right)=\rho_{t_{2}}\left(\tau_{g_{1}}(x)\right)=\rho_{t_{2}}\left(\rho_{t_{1}}(x)\right)=\rho_{t_{1}}\left(\rho_{t_{2}}(x)\right)=\rho_{t_{1} t_{2}}(x)$. Since $G_{[x]}$ is compact, the image of the map $A$ is a compact subgroup of $\mathbf{R}^{+}$ and is therefore the identity. Hence $A(g)=1$, for all $g \in G_{[x]}$ and $t=1$. The proof of (5) is a direct consequence of (4) and the standard analogous result for compact group actions on compact manifolds (see, for example, [4] and $[8])$.

From the proof of the last item, we conclude that the $\mathbf{R}^{+}$-orbits are transversal to the torus orbits. Notice that the description given for $\Phi$ is not invariant under translations, as is frequent for moment maps.

Let $T_{1}, \ldots, T_{K}$ be the subgroups of $T^{r}$ occurring as stabilizer groups of points of the symplectic cone $X$. Let $X_{i}=\{x \in X \mid$ the isotropy group of $x$ is $T_{i}$ \}. By relabeling, we can assume the $X_{i}$ 's to be connected. Then $X$ can be written as

$$
X=\sqcup_{i=1}^{N} X_{i} \quad \text { (disjoint union). }
$$

We now prove the analogous of Theorem 3.7 and 3.8 in [4].

Proposition 1.2. Each $X_{i}$ in the decomposition $X=\sqcup_{i=1}^{N} X_{i}$ is a $T^{r}$ invariant conic symplectic submanifold of $X$ of strictly positive dimension and $\Phi$ maps each $X_{i}$ submersively onto an open subset of $\left(\operatorname{Lie} T_{i}\right)^{\perp}$, the annihilator space of Lie $T_{i}$ in (Lie $\left.T_{i}\right)^{*}$. Moreover, $\Phi\left(X_{i}\right)$ is a union of a finite number of polytopic convex cones.

Proof. Since $T^{r}$ is abelian, $T^{r} \cdot X_{i} \subseteq X_{i}$. Since the actions of $\mathbf{R}^{+}$and $T^{r}$ commute and $\mathbf{R}^{+}$acts freely on $X, \mathbf{R}^{+}$also acts freely on $X_{i}$ and so $X_{i}$ is a conic manifold of non-zero dimension. By Theorem 3.5 in [4], $X_{i}$ is symplectic (and so even dimensional). The map $\Phi_{i}=\left.\Phi\right|_{X_{i}}$ is the moment map associated to the action of $T^{r}$ on $X_{i}$. Also as in [4], $\left(d \Phi_{i}\right)_{x}$ maps $T_{x} X_{i}$ onto (Lie $\left.T_{i}\right)^{\perp}$ for all $x \in X_{i}$ and hence $\Phi$ maps $X_{i}$ submersively onto an open subset of $p_{i}+\left(\operatorname{Lie} T_{i}\right)^{\perp}$ where $p_{i}=\Phi_{i}(x)$. From the homogeneity of $\Phi, \Phi\left(X_{i}\right)$ is an open cone of the vector space $\left(\operatorname{Lie} T_{i}\right)^{\perp}$.

Since $0 \notin \Phi(X), \Phi$ is homogeneous and $X_{i}$ is conic, we have that $0<$ $\operatorname{dim}\left(\Phi\left(X_{i}\right)\right)=\operatorname{dim}\left(\left(\operatorname{Lie} T_{i}\right)^{\perp}\right)$ - induction starts with $k=1$.

If the codimension of Lie $T_{i}$ is $1, \Phi\left(X_{i}\right)$ is an open and conic subset in the one-dimensional space $\left(\operatorname{Lie} T_{i}\right)^{\perp}$. Since $0 \notin \Phi(X), 0 \notin \Phi\left(X_{i}\right)$ and $\Phi\left(X_{i}\right)$ 
is an open half-line starting at 0 . Assume that the result is true when $\operatorname{codim}\left(\operatorname{Lie} T_{i}\right)<k$. If $\operatorname{codim}\left(\operatorname{Lie} T_{i}\right)=k, \Phi\left(X_{i}\right)$ is a $k$-dimensional open subset of the vector space $\left(\operatorname{Lie} T_{i}\right)^{\perp}$ and its boundary components, if they exist, are the connected components of $\cup_{j} \Phi\left(X_{j}\right), T_{j} \supset T_{i}$. By induction, each $\Phi\left(X_{j}\right)$ is a finite union of polytopic convex cones. Therefore the boundary of $\Phi\left(X_{i}\right)$ is a finite union of polytopic convex cones and so the same holds for $\Phi\left(X_{i}\right)$ itself.

We continue using the same techniques as in [4], but instead of inspecting the properties of the maps $\Phi^{\xi}: X \rightarrow \mathbf{R}, \xi \in$ Lie $T^{r}$, we consider the restrictions $\left.\Phi^{\xi}\right|_{M}: M \rightarrow \mathbf{R}, \xi \in \operatorname{Lie} T^{r}$, where $M=\Phi_{0}^{-1}(1)$, the compact submanifold of $X$ defined in Proposition 1.1.

Lemma 1.3. The function $\left.\Phi^{\xi}\right|_{M}: M \rightarrow \mathbf{R}$ has a unique local maximum.

Before proving this lemma, we use it to prove Theorem 1.

Proof of Theorem 1. We want to show that $\Phi(X)$ is a polytopic convex cone. By Proposition 1.2, it is enough to show that $\Phi(X)$ is convex.

Since $X=\rho_{t}(M)$ and the moment map satisfies $\Phi(X)=\Phi\left(\rho_{t}(M)\right)=$ $t \cdot \Phi(M), t \in \mathbf{R}^{+}$, we only need to show that $\Phi(M)$ is convex.

Let $p$ be a point in the boundary of $\Phi(M) \subset \Phi(X)$ and $m \in M$ a preimage of $p$. From the local form of the moment map (again, as in [4]), there is an open neighborhood $U$ of $m$ in $X$ taken to the intersection $U^{\prime}$ of a convex polytopic cone with vertex $p$ with an open neighborhood of $p$ in $\left(\operatorname{Lie} T^{r}\right)^{*}$. Consider now the affine hyperplane $H_{1}=\left\{v \in \mathbf{R}^{r} \mid\left\langle v, \xi_{0}\right\rangle=1\right\}$. Clearly, the restricted neighborhoods $V=U \cap M \subset M$ and $V=V^{\prime} \cap H_{1}$ satisfy $\Phi(V)=V^{\prime}$, where $V^{\prime}$ is also the intersection of a convex polytopic cone with vertex $p$ with a neighborhood of $p$ in $H_{1}$.

Let $S_{i}$ be a boundary component of $V^{\prime}$ containing $p$. We can choose $\xi \in \operatorname{Lie} T^{r}$ such that $\langle\xi, f\rangle=0$, for $f \in S_{i}$ and $\langle\xi, f\rangle<0$ in the interior of $V^{\prime}$.

Then $\Phi^{\xi}(y)=\langle\xi, \Phi(y)\rangle \leq 0$, for all $y \in V$. So, 0 is a local maximum of $\left.\Phi^{\xi}\right|_{M}$ and by Lemma $1.3,0$ is a global maximum of $\left.\Phi^{\xi}\right|_{M}$, i.e. $\Phi^{\xi}(M) \leq 0$.

Mimicking again the argument in $[4], \Phi(M)$ behaves as a convex set relative to its boundary points and must then be convex.

Lemma 1.3 follows from Morse theory as expanded for clean functions by Bott ([3], [4]). Let $K$ be a compact connected manifold of dimension $n$. Recall that, for a clean function $f: K \rightarrow \mathbf{R}$, the index (i.e., the number of strictly negative eigenvalues of the Hessian of $f$ at a point) is constant along a connected component $C_{i}$ of the critical set of $f$. Also, a clean function $f: K \rightarrow \mathbf{R}$ with indices on critical components different from 1 or $n-1$ has a unique local maximum. Lemma 1.3 is an immediate consequence of the theorem below. 
Proposition 1.4. The function $\left.\Phi^{\xi}\right|_{M}: M \rightarrow \mathbf{R}$ is clean. The indices of the critical manifolds of $\left.\Phi^{\xi}\right|_{M}$ and the number of positive eigenvalues of the Hessian at a critical point are even.

The proof of this result requires some preparation.

Lemma 1.5. $\Phi(X) \cup\{0\}$ is closed in $\mathbf{R}^{r}$.

Proof. Suppose that there is a sequence $\left\{\Phi\left(x_{n}\right)\right\}$ converging to $v \in \mathbf{R}^{r}$. We need to show that $v \in \Phi(X) \cup\{0\}$. It is enough to consider $v \neq 0$. Since $\Phi\left(x_{n}\right) \rightarrow v$, there exists a sequence $\left\{\left(m_{n}, t_{n}\right)\right\} \in M \times \mathbf{R}^{+}$such that $\Phi\left(m_{n}, t_{n}\right) \rightarrow v$. But $\Phi\left(m_{n}, t_{n}\right)=t_{n} \Phi\left(m_{n}, 1\right)$, so $\left|t_{n}\right|\left\|\Phi\left(m_{n}, 1\right)\right\| \rightarrow\|v\|$. Since $0 \notin \Phi(X)$ and $\Phi\left(m_{n}, 1\right)$ is in a compact set, $\left\|\Phi\left(m_{n}, 1\right)\right\|$ is bounded away from zero and so $t_{n}$ is in a in a compact subset of $\mathbf{R}^{+}$. Then the sequence $\left\{x_{n}\right\}=\left\{\left(m_{n}, t_{n}\right)\right\}$ has a convergent subsequence in $X$ and $0 \neq v=$ $\lim _{n \rightarrow \infty} \Phi\left(x_{n}\right) \in \Phi(X)$.

Because of Lemma 1.5, $\left\{x \mid\left\langle x, \xi_{0}\right\rangle>0\right\} \backslash \Phi(X)$ has a non-empty interior, so we can assume (after a small perturbation if necessary) that $\xi_{0}$ is in the set $\frac{1}{m} \mathbf{Z}^{r}$ for some integer $m$ (here, $\mathbf{Z}^{r}$ is the kernel of exp : Lie $T^{r} \rightarrow T^{r}$ ). Thus $\xi_{0}$ generates an action of the circle group $S^{1}$ on $X$ with moment map $\Phi_{0}=\left\langle\Phi, \xi_{0}\right\rangle: X \rightarrow \mathbf{R}^{+}$. Since $0<\left\langle\Phi, \xi_{0}\right\rangle=\Phi_{0}=\iota\left(\xi_{0}^{\sharp}\right) \alpha$, the $S^{1}-$ action is locally free.

The proof of Proposition 1.4 makes use of a normal form for the moment map of a torus action in a neighborhood of a point. This problem has been solved by Guillemin and Sternberg in [5] and by Marle in [8]. We now outline the construction in Lerman [7], from which the results in [5] and [8] obtain the requested information. Let $(X, \omega)$ be a $2 n$-dimensional symplectic manifold on which the torus $T^{r}$ acts in Hamiltonian fashion and let $\Phi_{X}: X \rightarrow \mathbf{R}^{r}$ be the associated moment mapping. Let $Z$ be the action orbit through $x$ and consider the (symplectic) vector space $V=T Z_{x}^{\perp} / T Z_{x}$, where the orthogonality sign is to be understood with respect to $\omega$ at the point $x$. For $x \in X$, let $T_{x} \subseteq T^{r}$ be its isotropy group, and denote its connected component containing the identity by $T_{x}^{o}$. Finally, let $K$ be a subtorus such that $T^{r}=K \times T_{x}^{o}$. (Since we are interested strictly in a local normal form at $x$, the possible existence of other connected components of the isotropy group is irrelevant, but both cases are treated in [7].) Consider now the model symplectic manifold $Y=T^{*} K \times V$ on which $T^{r}=K \times T_{x}^{o}$ acts as a product of a free (right) action of $K$ in the cotangent bundle $T^{*} K$ and a linear symplectic action of $T_{x}^{o}$ in $V$. Denote by $\Phi_{Y}$ the moment map of this action. From [5] or [8], in an appropriate $T^{r}$-invariant neighborhood of $x$ in $X$, there is a symplectic change of coordinates $\phi$ to a neighborhood 
of the origin of $T^{*} K \times V$ taking one torus action to another. In particular, the moment maps $\Phi_{X}$ and $\Phi_{Y}$ are related by $\Phi_{X}=\Phi_{Y} \circ \phi$. Also, it is simple to obtain variables so that

$$
\begin{aligned}
& \Phi\left(x_{1}, y_{1}, \ldots, x_{k}, y_{k}, x_{k+1}, y_{k+1}, \ldots, x_{n}, y_{n}\right) \\
& =e_{1} y_{1} \ldots+e_{k}\left(y_{k}+1\right)+\alpha_{k+1}\left(x_{k+1}^{2}+y_{k+1}^{2}\right) / 2+\ldots+\alpha_{n}\left(x_{n}^{2}+y_{n}^{2}\right) / 2,
\end{aligned}
$$

where the $e_{i}$ 's are canonical vectors and the $\alpha_{j}$ 's are weights of the linearization of the action of the isotropy $T_{x}$ on $V$, following the usual procedures for normal forms as in [6] (also [5]). The subspaces spanned by $\left\{\alpha_{1}, \ldots, \alpha_{k}\right\}$ and $\left\{e_{k+1}, \ldots, e_{n}\right\}$ have trivial intersection. Notice that the coordinate system can be taken so that the moment map $\Phi_{0}$ corresponding to the locally free $S^{1}$-action is $\Phi_{0}=y_{k}+1$.

Proof of Proposition 1.4. Let $C_{M}$ be the critical set of $\left.\Phi^{\xi}\right|_{M}$. It suffices to show that the Hessian of $\left.\Phi^{\xi}\right|_{M}$ at $x \in C_{M}$ has an even number of positive (negative) eigenvalues and that its kernel at $x$ is the tangent space of $C_{M}$ at $x$. Clearly, it suffices to check both statements for a convenient coordinate system - we consider $\Phi$ in the local normal form described above on a neighborhood $\left\{x_{1}, y_{1}, \ldots, x_{n}, y_{n}\right\}$ of the origin. For points in $M$ near the origin, we must have $y_{k}=0$. A simple computation shows that, locally, the critical component containing zero is a subspace. Also, for the nondegenerate part of the Hessian, the double partial derivatives in variables $x_{j}$ and $y_{j}$, for $j=k, \ldots, n$, give rise to eigenvalues of the same sign.

\section{A Rigidity theorem.}

Let $(X, \omega)$ be a symplectic cone with compact base, admitting a symplectic $T^{r}$ action which commutes with the $\mathbf{R}^{+}$-action. We can assume, without loss, that $T^{r}$ acts locally freely on a conic open subset $X^{\prime}$ of $X$ and that the complement of $X^{\prime}$ is of codimension 2 in $X$.

Let $\Phi:(X, \omega) \rightarrow \mathbf{R}^{r}$ be the moment map associated to the $T^{r}$-action. Assume that $\left\langle\Phi, \xi_{0}\right\rangle>0$ for some $\xi_{0} \in \frac{1}{m} \mathbf{Z}^{r}$, for some integer $m$, as remarked after Lemma 1.5. By Theorem $1, \Phi(X)$ is a polytopic convex cone.

Consider now a symplectic form $\Omega$ on $X$, not necessarily satisfying $\rho_{t}^{*} \Omega=$ $t \cdot \Omega$, but such that the same $T^{r}$-action on $X$ admits a moment map $\Psi$ with respect to $\Omega$.

We make the following assumptions about $\Psi$.

1. $\lim _{t \rightarrow 0} \Psi(t, m)=0$ uniformly in $m \in M$. (This is equivalent to saying that the contraction of $\Omega-\omega$ with any vector field induced by the torus action is integrable close to the vertex of $X, t=0$.)

2. $\lim _{t \rightarrow \infty} \Psi_{0}(t, m)=\infty$, where $\Psi_{0}=\left\langle\Psi, \xi_{0}\right\rangle$, uniformly in $m \in M$. 
We will show that under these assumptions, the following rigidity theorem holds.

Theorem 2. The images of $\Phi$ and $\Psi$ coincide.

Corollary. Let $\Phi:(X, \omega) \rightarrow \mathbf{R}^{r}$ and $\Psi:(X, \Omega) \rightarrow \mathbf{R}^{r}$ be moment maps as above. Suppose also that $0 \notin \Phi(X)$ and that there exists a $\xi_{0} \in \mathbf{R}^{r}$ such that $\left\langle\Phi, \xi_{0}\right\rangle>0$ on $X$. If $\Omega-\omega$ is compactly supported, then the images of $\Phi$ and $\Psi$ coincide up to a translation.

In the notation of Section 1, $X$ can be written as a disjoint union $X=$ $\sqcup_{i=1}^{N} X_{i}$, where $X_{i}=\left\{x \in X \mid\right.$ the stabilizer of $x$ is $\left.T_{i}\right\}$ is connected and $\Phi(X)=\cup_{i=1}^{N} \Phi\left(X_{i}\right), \Psi(X)=\cup_{i=1}^{N} \Psi\left(X_{i}\right)$. The boundaries of both moment maps are formed by faces of the form $\Phi\left(X_{k}\right)$ or $\Psi\left(X_{k}\right)$, with $T_{k}$ different from the identity. On the other hand, some (interior) faces do not lie on the boundaries of the images, but in the interiors of the images - they will receive special attention in the proof of Theorem 2 .

We will need some preparatory lemmas.

Lemma 2.1. $0 \notin \Psi(X)$.

Proof. Consider $\Psi_{0}(x)=\left\langle\xi_{0}, \Psi(x)\right\rangle, x \in X$. We first show that $\Psi_{0}$ has an open image. This follows from the simple fact below, together with the openness of $\left.\Psi\right|_{X_{i}}$ in $\left(\operatorname{Lie} T_{i}\right)^{\perp}$.

Fact: $\left.\left\langle\xi_{0}, \cdot\right\rangle\right|_{\left(\operatorname{Lie} T_{i}\right)^{\perp}}$ is not identically zero for any $\left(\operatorname{Lie} T_{i}\right)^{\perp}$.

Proof of fact.

If $\left\langle\xi_{0},\left.\cdot\right|_{\left(\operatorname{Lie} T_{i}\right)^{\perp}}\right.$ were identically zero, $\left(\operatorname{Lie} T_{i}\right)^{\perp}$ would be contained in the hyperplane $H_{0}=\left\{v \in\left(\operatorname{Lie} T^{r}\right)^{*} \mid\left\langle\xi_{0}, v\right\rangle=0\right\}$ (since the fact that $\Phi(X)$ has no vertices implies that $\left.\operatorname{dim}\left(\operatorname{Lie} T_{i}\right)^{\perp} \geq 1\right)$. But $\Phi\left(X_{i}\right) \subset\left(\operatorname{Lie} T_{i}\right)^{\perp}$ and, by hypothesis, $\Phi(X)$ and so $\Phi\left(X_{i}\right)$ are contained in the interior of $H_{+}=$ $\left\{v \in\left(\operatorname{Lie} T^{r}\right)^{*} \mid\left\langle\xi_{0}, v\right\rangle>0\right\}$. Hence $\left.\left\langle\xi_{0}, \cdot\right\rangle\right|_{\left(\operatorname{Lie} T_{i}\right)^{\perp}}$ cannot be identically zero.

Now, notice that $\Psi_{0}$ is bounded from below. Indeed, suppose it is not. Then there would be a sequence $\left\{x_{n}\right\}$ in $X$ such that $\lim _{n \rightarrow \infty} \Psi_{0}\left(x_{n}\right)=$ $-\infty$. The sequence would not converge in $X$, for if it did, $\left\{\Psi_{0}\left(x_{n}\right)\right\}$ would converge too. Since we can write $x_{n}=\left(m_{n}, t_{n}\right)$, with $m_{n} \in M$, we have two possibilities: For a subsequence, either $t_{n} \rightarrow \infty$ and $\Psi_{0}\left(x_{n}\right) \rightarrow \infty$ (by hypothesis) or $t_{n} \rightarrow 0$ and $\Psi_{0}\left(x_{n}\right) \rightarrow 0$ (by hypothesis). Hence we never have $\Psi_{0}\left(x_{n}\right) \rightarrow-\infty$ and $\Psi_{0}$ is bounded from below.

If $0 \in \Psi(X), \Psi_{0}$ attains a negative value $-\epsilon$ by the openness of its image and by using the hypothesis at the extremes of the cone again, the set 
$\Psi_{0}^{-1}(-\infty,-\epsilon / 2]$ is contained in a compact set of the form $M \times[a, b], 0<$ $a<b<\infty$. Then $\Psi_{0}$ attains its infimum, contradicting the openness of its image.

In the next lemma we will show that the map $\Phi$ is open at points that are taken into the interior faces. In Lemma 2.3 we will show an analogous result for $\Psi$.

Lemma 2.2. Assume that there exists $x_{k}$ in $X_{k}$ such that $\Phi\left(x_{k}\right) \in$ Interior $(\Phi(X))$. Then $\Phi$ is an open map at the point $x_{k}$ - i.e., there is an open neighborhood of $x_{k}$ in $X$ taken to an open neighborhood of $\Phi\left(x_{k}\right)$ in $\left(\operatorname{Lie} T^{r}\right)^{*}$. Similarly, $\Psi$ is also open at $x_{k}$.

Proof. By the usual local convexity of the moment map as in [4], the image of a sufficiently small neighborhood $U$ of $x_{k}$ is taken to an intersection of halfspaces whose bounding hyperplanes contain $\Phi\left(x_{k}\right)$, which without loss in this argument we assume to be the origin of $\left(\operatorname{Lie} T^{r}\right)^{*}$. Let one such bounding hyperplane be the annihilator of some $\xi \in \operatorname{Lie} T^{r}$ and orient $\xi$ so that the half-space containing $\Pi(U)$ is of the form $\langle\xi, f\rangle \leq 0$, for $f \in\left(\operatorname{Lie} T^{r}\right)^{*}$. By Lemma 1.3, the local maximum $x_{k}$ of the function $\left.\Phi^{\xi}\right|_{M}$ is a point in which $\left.\Phi^{\xi}\right|_{M}$ attains its maximum, contradicting the fact that $\Phi\left(x_{k}\right)$ is an interior point of $\Phi(X)$. Thus, there can be no such bounding hyperplanes and $\Phi$ is open at $x_{k}$. Moreover, from the local form of $\Phi$ at $x_{k}$, we have that the weights $\alpha_{1}, \ldots, \alpha_{n}$ of the linear isotropy action of $T_{k}$ on $\left(T_{x} X, \omega_{x_{k}}\right)$ span $\left(\operatorname{Lie} T^{r}\right)^{*}$. Now, $T_{k}$ induces the same (linear) action on the vector space $T_{x} X$, which can be interpreted in two different ways as a symplectic action, depending if the bilinear form considered in $T_{x} X$ is $\omega_{x_{k}}$ or $\Omega_{x_{k}}$. By Darboux theorem, both actions are conjugate by a symplectic transformation and since the weights of the first action span $\left(\operatorname{Lie} T^{r}\right)^{*}$, the same must hold for the weights of the second action, implying the openness of $\Psi$ at $x_{k}$.

We now start the proof of Theorem 2.

We first prove that $\Psi\left(\overline{X_{i}}\right) \subseteq \Phi\left(\overline{X_{i}}\right)$ (where $\overline{X_{i}}$ is the closure of $X_{i}$ in $X$ and so $\Phi\left(\overline{X_{i}}\right)$ does not contain 0$)$ and conclude that $\Psi(X) \subseteq \Phi(X)$. Then we prove that $\Psi(X)$ covers an open dense set of $\Phi(X)$ and by taking closures we conclude that both images are equal.

Step 1. $\Psi\left(\overline{X_{i}}\right) \subseteq \Phi\left(\overline{X_{i}}\right)$.

Proof. The proof will be by induction on $k=\operatorname{dim}\left(\operatorname{Lie} T_{i}\right)^{\perp}$, which is the dimension of the interior of either $\Psi\left(\overline{X_{i}}\right)$ or $\Phi\left(\overline{X_{i}}\right)$.

As in the proof of Proposition 1.2, $k \geq 1$. From Theorem 1, for $\Phi(X)$, there exists $X_{i}$ such that the interior of $\Phi\left(\overline{X_{i}}\right)$ has dimension equal to 1 .

If $k=1$, then $\Phi\left(\overline{X_{i}}\right)$ is an open half-line starting at 0 , contained in the straight line $\left(\operatorname{Lie} T_{i}\right)^{\perp}$, and $\Psi\left(\overline{X_{i}}\right)$ is an open subset in $\left(\operatorname{Lie} T_{i}\right)^{\perp}+p$, for some 
$p \in \mathbf{R}^{r}$. Now, $\overline{X_{i}}$ is a conic manifold, so $\Psi\left(\rho_{t}(x)\right) \in \Psi\left(\overline{X_{i}}\right)$, for positive $t$ and $x \in \overline{X_{i}}$. By hypothesis, $\lim _{t \rightarrow 0} \Psi\left(\rho_{t}(x)\right)=0$, so $\Psi\left(\overline{X_{i}}\right)$ is also a subset of $\left(\operatorname{Lie} T_{i}\right)^{\perp}$. Moreover, $0 \notin \Psi(X)$ by Lemma 2.1, (hence $0 \notin \Psi\left(\overline{X_{i}}\right)$ ), and the limits along the fiber $\rho_{t}(x)$ are equal to 0 and $\infty$ by hypothesis. So we must have that the connected set $\Psi\left(\overline{X_{i}}\right)$, which is open in $\left(\operatorname{Lie} T_{i}\right)^{\perp}$, is also an open half-line beginning at 0 contained in $\left(\operatorname{Lie} T_{i}\right)^{\perp}$. The case $k=1$ is proved.

Suppose now that $\Psi\left(\overline{X_{i}}\right) \subseteq \Phi\left(\overline{X_{i}}\right)$ for all $X_{i}$ such that $\operatorname{dim}\left(\operatorname{Lie} T_{i}\right)^{\perp}<k$. We will show that the same is true for all $\overline{X_{i}}$ such that $\operatorname{dim}\left(\operatorname{Lie} T_{i}\right)^{\perp}=k$.

As shown in Section 27 of [6], $\overline{X_{i}}$ is a symplectic manifold, hence a symplectic cone with compact base and $\Phi\left(\overline{X_{i}}\right)$ is a polytopic convex cone in $\left(\operatorname{Lie} T_{i}\right)^{\perp}$ (Theorem 1).

Set $A=\Phi\left(\overline{X_{i}}\right) \cup\{0\}$ and $B=A^{c}$, the complement of $A$ in $\left(\operatorname{Lie} T_{i}\right)^{\perp}$ which is open by Lemma 1.5. We first show that $\Psi\left(\overline{X_{i}}\right) \cap B=B$ or the empty set, using a connectedness argument.

i) $\Psi\left(\overline{X_{i}}\right) \cap B$ is open in $B$.

$\Psi\left(\overline{X_{i}}\right)=\Psi\left(X_{i}\right) \cup\left(\cup_{j} \Psi\left(\overline{X_{j}}\right)\right)$, where $T_{j} \supset T_{i}$, and by induction $\Psi\left(\overline{X_{j}}\right) \subseteq$ $\Phi\left(\overline{X_{j}}\right) \subset A$. So $\Psi\left(\overline{X_{i}}\right) \cap B=\Psi\left(X_{i}\right) \cap B$ and $\Psi\left(\overline{X_{i}}\right) \cap B$ is open since both $\Psi\left(X_{i}\right)$ and $B$ are.

ii) $\Psi\left(\overline{X_{i}}\right) \cap B$ is closed in $B$.

Consider a sequence $\left\{y_{n}=\Psi\left(x_{n}\right)\right\}$ in $\Psi\left(\overline{X_{i}}\right) \cap B$ converging to $y \in B$. Since $X \simeq M \times \mathbf{R}^{+}$, we can write $x_{n}=\left(m_{n}, t_{n}\right)$. Since $\left\{y_{n}\right\}$ converges to $y \in B$ and $0 \notin B, t_{n}$ is far from zero and from $\infty$. Hence the sequence $\left\{x_{n}\right\}$ is in a compact set in $X$ and admits a subsequence converging to a point $x \in \overline{X_{i}}$. Therefore $\left\{y_{n}\right\}$ converges to $y=\Psi(x) \in \Psi\left(\overline{X_{i}}\right)$ and $\Psi\left(\overline{X_{i}}\right) \cap B$ is closed in $B$.

Since $B$ is connected, $\Psi\left(\overline{X_{i}}\right) \cap B=B$ or $\Psi\left(\overline{X_{i}}\right) \cap B=\emptyset$. But $\Psi_{0}(X)$ is bounded from below (by the proof of Lemma 2.1) so we cannot have $\Psi\left(\overline{X_{i}}\right) \cap B=B$. Therefore $\Psi\left(\overline{X_{i}}\right) \subseteq A=\Phi\left(\overline{X_{i}}\right) \cup\{0\}$. Since $0 \notin \Psi(X), 0 \notin$ $\Psi\left(\overline{X_{i}}\right)$ and then $\Psi\left(\overline{X_{i}}\right) \subseteq \Phi\left(\overline{X_{i}}\right)$, completing the proof of Step 1 .

In particular, $\Psi(X) \subseteq \Phi(X)$.

We now show that $\Psi(X)$ covers an open dense set of $\Phi(X)$.

Let $X_{m}=\{x \in X \mid$ the isotropy group of $x$ is the identity $\}$. By Section 3 of [4], $X_{m}$ is a connected open set in $X$.

Consider the set

$$
C=\left\{\Psi\left(\overline{X_{i}}\right) \text { for } X_{i} \neq X_{m} \mid \Psi\left(\overline{X_{i}}\right) \subset \operatorname{Int}(\Phi(X))\right\} .
$$

Recall that for all $X_{i}$ in the stratification $X=\sqcup_{i=1}^{N} X_{i}, \Psi\left(\overline{X_{i}}\right) \subseteq \Phi\left(\overline{X_{i}}\right) \subset$ $\Phi(X)$. 
Let $\tilde{C}=\operatorname{Int}(\Phi(X))-C$, which is clearly an open set, and $A_{1}, \ldots, A_{s}$ be the open connected components of $\tilde{C}$.

Step 2. $\Psi(X) \cap A_{j} \neq \emptyset \quad j=1, \ldots, s$.

Proof. If $s=1, \tilde{C}$ has only one connected component $A_{1}$. Since $\Psi(X) \subseteq$ $\Phi(X)$, the result follows in this case. Consider now $s>1$. For each $A_{j}$, there exists at least one $\Psi\left(\overline{X_{i}}\right) \in C$ such that $\Psi\left(\overline{X_{i}}\right)$ is in the boundary of $A_{j}$. By Lemma 2.2, given $x \in X_{i}$ such that $\Phi(x) \in \operatorname{Int}(\Phi(X))$, there exists an open set $V_{w}^{\prime}$ of $\Psi(X)$ around $w=\Psi(x)$ in $\left(\operatorname{Lie} T^{r}\right)^{*}$. So $V_{w}^{\prime}$ intersects all the $A_{j}$ 's that have $\Psi\left(\overline{X_{i}}\right)$ as a boundary component and then $V_{w}^{\prime} \cap A_{j} \neq \emptyset$ and $\Psi(X) \cap A_{j} \neq \emptyset$. We conclude that for any $A_{j}$, there is an open set in $\Psi(X)$ that intersects it. Hence $\Psi(X) \cap A_{j} \neq \emptyset$ for $j=1, \ldots, s$.

Step 3. $\Psi(X) \cap A_{j}=A_{j}, \quad j=1, \ldots, s$.

Proof. This is connectedness again.

i) $\Psi(X) \cap A_{j}$ is open in $A_{j}$.

Since $\Psi(X)=\Psi\left(X_{m}\right) \cup\left(\cup_{i} \Psi\left(\overline{X_{i}}\right)\right), \Psi(X) \cap A_{j}=\Psi\left(X_{m}\right) \cap A_{j}$. Therefore, $\Psi(X) \cap A_{j}$ is open, since both $\Psi\left(X_{m}\right)$ and $A_{j}$ are.

ii) $\Psi(X) \cap A_{j}$ is closed in $A_{j}$.

Let $\left\{y_{n}=\Phi\left(x_{n}\right)\right\}$ be a sequence in $\Psi(X) \cap A_{j}$ converging to $y \in A_{j}$. We want to show that $y \in \Psi(X) \cap A_{j}$, i.e., that $y \in \Psi(X)$. We now make use of compactness as above. Again, since $y \neq 0,\left\{x_{n}\right\}$ has a subsequence that converges in $X$. Then $\left\{y_{n}\right\}$ converges to $y \in \Psi(X)$.

Therefore $\Psi(X) \cap A_{j}$ is not empty (from Step 2), open and closed in the connected set $A_{j}$, and so $\Psi(X) \cap A_{j}=A_{j}$ and $A_{j} \subseteq \Psi(X)$. We conclude then that $\Psi(X)$ covers an open dense subset of $\Phi(X)$ since $\operatorname{Int}(\Phi(X))$ $C=\cup_{j} A_{j} \subseteq \Psi(X)$. The closure of $\operatorname{Int}(\Phi(X))-C$ is $\Phi(X) \cup\{0\}$ and from a by now standard compactness argument the closure of $\Psi(X)$ is also its union with $\{0\}$. So, taking closures, $\Phi(X) \cup\{0\} \subseteq \Psi(X) \cup\{0\}$. Since $0 \notin \Phi(X)$ and $0 \notin \Psi(X)$ we conclude that $\Phi(X) \subset \Psi(X)$. By Step 1, $\Psi(X)=\Phi(X)$. 


\section{Appendix.}

Example 1. Let $X=\mathbf{R}^{2 n}-\{0\}$ with symplectic form $\omega=\sum_{i=1}^{n} d x_{i} \wedge d y_{i}$ and

$$
\rho_{t}\left(x_{1}, y_{1}, \ldots, x_{n}, y_{n}\right)=\left(\sqrt{t} x_{1}, \sqrt{t} y_{1}, \ldots, \sqrt{t} x_{n}, \sqrt{t} y_{n}\right) t \in \mathbf{R}^{+}
$$

an action of $\mathbf{R}^{+}$on $X$. It is easy to see that $X / \mathbf{R}^{+}=S^{2 n-1}$ and that $\left(\rho_{t}\right)^{*} \omega=$ $t \cdot \omega$. Hence $(X, \omega)$ with this $\mathbf{R}^{+}$-action is a symplectic cone.

Consider the following $T^{n}$-action on $X$ :

$$
\tau_{\left(\alpha_{1}, \ldots, \alpha_{n}\right)}\left(x_{1}, y_{1}, \ldots, x_{n}, y_{n}\right)=\left(\begin{array}{cccc}
\cos \alpha_{1} & -\operatorname{sen} \alpha_{1} & & \\
\operatorname{sen} \alpha_{1} & \cos \alpha_{1} & & \\
& & \ddots & \\
& & \cos \alpha_{n} & -\operatorname{sen} \alpha_{n} \\
& & \operatorname{sen} \alpha_{n} & \cos \alpha_{n}
\end{array}\right)\left(\begin{array}{c}
x_{1} \\
y_{1} \\
\vdots \\
x_{n} \\
y_{n}
\end{array}\right)
$$

It is clear that $\tau \circ \rho=\rho \circ \tau$. Moreover, this $T^{n}$-action is Hamiltonian with moment map $\Phi: X \rightarrow \mathbf{R}^{n}$, where

$$
\Phi\left(x_{1}, y_{1}, \ldots, x_{n}, y_{n}\right)=\left(\frac{x_{1}^{2}+y_{1}^{2}}{2}, \ldots, \frac{x_{n}^{2}+y_{n}^{2}}{2}\right)
$$

and $\operatorname{Im} \Phi=\left\{\left(r_{1}, \ldots, r_{n}\right) \in \mathbf{R}^{n}-\{0\} \mid r_{i} \geq 0 \quad i=1, \ldots, n\right\}$.

Let $\Omega$ be a $T^{n}$-invariant symplectic form in $X$ obtained by a perturbation of $\omega$, i.e., $\Omega=\omega+\mu$ where $\mu$ is a closed 2 -form and $\Omega$ is non-degenerate. It is possible to show that the most general $\Omega$ satisfying these properties is given by

$$
\begin{aligned}
\Omega= & \omega \\
& +\sum_{i} c_{i i} d x_{i} \wedge d y_{i} \\
& +\sum_{i<j}\left(a_{i j} x_{i} x_{j}-c_{i j} \frac{x_{i} y_{j}}{x_{j}^{2}+y_{j}^{2}}+c_{j i} \frac{x_{j} y_{i}}{x_{i}^{2}+y_{i}^{2}}\right) d x_{i} \wedge d x_{j} \\
& +\sum_{i<j}\left(a_{i j} x_{i} y_{j}+c_{i j} \frac{x_{i} x_{j}}{x_{j}^{2}+y_{j}^{2}}+c_{j i} \frac{y_{i} y_{j}}{x_{i}^{2}+y_{i}^{2}}\right) d x_{i} \wedge d y_{j} \\
& +\sum_{i<j}\left(-a_{i j} x_{j} y_{i}+c_{i j} \frac{y_{i} y_{j}}{x_{j}^{2}+y_{j}^{2}}+c_{j i} \frac{x_{i} x_{j}}{x_{i}^{2}+y_{i}^{2}}\right) d x_{j} \wedge d y_{i} \\
& +\sum_{i<j}\left(a_{i j} y_{i} y_{j}+c_{i j} \frac{x_{j} y_{i}}{x_{j}^{2}+y_{j}^{2}}-c_{j i} \frac{x_{i} y_{j}}{x_{i}^{2}+y_{i}^{2}}\right) d y_{i} \wedge d y_{j}
\end{aligned}
$$

where, if $u_{i}=\frac{x_{i}^{2}+y_{i}^{2}}{2}$, 
1) $c_{i j}=u_{j} \gamma_{i j}\left(u_{1}, \ldots, u_{n}\right)$ with $\gamma_{i j} \in C^{\infty}\left(\mathbf{R}^{n}-\{0\}\right)$ and $\left(c_{i j}\right)_{u_{k}}=\left(c_{k j}\right)_{u_{i}}$ $\forall i \neq k, i \neq j$

2) $a_{i j}$ is a function of $u_{1}, \ldots, u_{n}$ and $\left(a_{i j}\right)_{u_{k}}-\left(a_{k j}\right)_{u_{i}}+\left(a_{i k}\right)_{u_{j}} \neq 0 \quad \forall i \neq j \neq k$ with $\Omega$ being non-degenerate.

The formulae in (1) and (2) must be interpreted in the variables $x_{i}, y_{i}$.

It is easy to see that $\Omega$ does not necessarily satisfy $\left(\rho_{t}\right)^{*} \Omega=t \cdot \Omega$. Let us check that $\Omega$ satisfies the properties above, i.e., it is $T^{n}$-invariant and closed. First, we will write $\Omega$ in a simpler form in $Y$, an open and dense subset of $X$, to be defined below.

Let $u_{i}=\frac{x_{i}^{2}+y_{i}^{2}}{2}, \theta_{i}=\operatorname{arctg} \frac{y_{i}}{x_{i}}$ and $Y=\left\{\left(u_{i}, \theta_{i}\right) i=1, \ldots, n, u_{i}>0\right\}$.

Notice that, by the inclusion of $Y$ in $X$, every $\Omega$ in $X$ gives rise to a form $\Omega^{*}$ in $Y$ but a form $\Omega^{*}$ in $Y$ is not naturally associated to an extension $\Omega$ in $X$. For this, it is necessary (and sufficient) that the coefficients $c_{i j}$ and $a_{i j}$ when written in the coordinates $\left(x_{i}, y_{i}\right)$ have a smooth extension from $Y$ to $X$.

Rewriting $\Omega$ in polar coordinates (in $Y$ ) we get:

$$
\Omega^{*}=\sum_{i} d u_{i} \wedge d \theta_{i}+\sum_{i} c_{i i} d u_{i} \wedge d \theta_{i}+\sum_{i \neq j} c_{i j} d u_{i} \wedge d \theta_{j}+\sum_{i<j} a_{i j} d u_{i} \wedge d u_{j} .
$$

The action of $T^{n}$ on $Y$ is given by:

$$
\tau_{\left(\alpha_{1}, \ldots, \alpha_{n}\right)}\left(u_{1}, \ldots, u_{n}, \theta_{1}, \ldots, \theta_{n}\right)=\left(u_{1}, \ldots, u_{n}, \theta_{1}+\alpha_{1}, \ldots, \theta_{n}+\alpha_{n}\right) .
$$

Since $c_{i j}$ and $a_{i j}$ are functions of the $u_{i}$ 's only, $\Omega^{*}$ is $T^{n}$-invariant in $Y$. Moreover, by the relations (1) and (2), we get that $\Omega^{*}$ is closed in $Y$.

The fact that $\Omega^{*}$ is closed and invariant by $\tau$ in $Y$ implies in closure and $\tau$-invariance of $\Omega$ in $X$ if the coefficients of $\Omega^{*}$ have a smooth continuation in $X$, and this is assured by (1) and (2). Now, writing $\Omega^{*}$ in matrix form we get:

$$
A_{\Omega^{*}}=\frac{1}{2}\left(\begin{array}{cccccccc}
0 & a_{12} & \ldots & a_{1 n} & 1+c_{11} & c_{12} & \ldots & c_{1 n} \\
-a_{12} & 0 & \ldots & a_{2 n} & c_{21} & 1+c_{22} & \ldots & c_{2 n} \\
\vdots & \vdots & & \vdots & \vdots & \vdots & & \vdots \\
-a_{1 n} & -a_{2 n} & \ldots & 0 & c_{n 1} & c_{n 2} & \ldots & 1+c_{n n} \\
& & & & & & & \\
-1-c_{11} & -c_{21} & \ldots & -c_{n 1} & 0 & 0 & \ldots & 0 \\
-c_{12} & -1-c_{22} & \ldots & -c_{n 2} & 0 & 0 & \ldots & 0 \\
\vdots & \vdots & & \vdots & \vdots & \vdots & & \vdots \\
-c_{1 n} & -c_{2 n} & \ldots & -1-c_{n n} & 0 & 0 & \ldots & 0
\end{array}\right) .
$$

We have that $\Omega^{*}$ is non-degenerate $\Longleftrightarrow \operatorname{det}\left(A_{\Omega^{*}}\right) \neq 0$. Therefore, in order for $\Omega$ to be non-degenerate, the coefficients $c_{i j}$ 's must satisfy some relations 
that must also hold in the limit when the $u_{i}$ 's go to 0 (not all of them simultaneously).

Let $\Psi: X \rightarrow \mathbf{R}^{n}$ be the moment map associated to the action of $\tau$ of $T^{n}$ on $(X, \Omega)$. It is easy to see that if $\Psi=\left(\Psi_{1}, \ldots, \Psi_{n}\right)$ then

$$
d \Psi_{k}=\left(1+c_{k k}\right) d u_{k}+\sum_{j \neq k} c_{j k} d u_{j} \quad k=1, \ldots, n .
$$

Let us look at some examples of $\Omega$ and $\Psi$ for $X=\mathbf{R}^{4}-\{0\}$ and compare the images of $\Phi$ and $\Psi$.

In this case, the non-degeneracy of $\Omega$ is equivalent to

$$
\operatorname{det}\left(\begin{array}{cccc}
0 & a_{12} & 1+c_{11} & c_{12} \\
-a_{12} & 0 & c_{21} & 1+c_{22} \\
-1-c_{11} & -c_{21} & 0 & 0 \\
-c_{12} & -1-c_{22} & 0 & 0
\end{array}\right) \neq 0 \text { in } \mathbf{R}^{4}-\{0\}
$$

that is, $1+c_{11}+c_{22}+c_{11} c_{22}-c_{12} c_{21} \neq 0, \quad \forall u_{1}, u_{2} \neq 0$ and

$$
\begin{aligned}
& \lim _{u_{1} \rightarrow 0}\left(1+c_{11}+c_{22}+c_{11} c_{22}-c_{12} c_{21}\right) \neq 0, \\
& \lim _{u_{2} \rightarrow 0}\left(1+c_{11}+c_{22}+c_{11} c_{22}-c_{12} c_{21}\right) \neq 0 .
\end{aligned}
$$

Example 1.1. Let $X=\mathbf{R}^{4}-\{0\}$ and $\Omega=\omega+\frac{\left(x_{1}^{2}+y_{1}^{2}\right)}{2} d x_{1} \wedge d y_{1}+\frac{\left(x_{2}^{2}+y_{2}^{2}\right)}{2} d x_{2} \wedge$ $d y_{2}$. The 2-form $\Omega$ is non-degenerate in $X$ since $1+c_{11}+c_{22}+c_{11} c_{22}-c_{12} c_{21}=$ $1+u_{1}+u_{2}+u_{1} u_{2} \neq 0$. Let $\Psi=\left(u_{1}+\frac{u_{1}^{2}}{2}, u_{2}+\frac{u_{2}^{2}}{2}\right)$. It is easy to see that

$$
\operatorname{Image}(\Psi)=\operatorname{Image}(\Phi)=\left\{(x, y) \in \mathbf{R}^{2}-\{0\} \mid x, y \geq 0\right\} .
$$

Note that the perturbation $\mu=\Omega-\omega$ satisfies $\left(\rho_{t}\right)^{*} \mu=t^{2} \mu$ and $\lim _{t \rightarrow 0} \Psi \circ$ $\rho_{t}\left(u_{1}, u_{2}\right)=(0,0)$.

Example 1.2. Let $X=\mathbf{R}^{4}-\{0\}$ and

$$
\begin{aligned}
& \Omega= \omega+2 \frac{\left(x_{2}^{2}+y_{2}^{2}\right)}{\left(x_{1}^{2}+y_{1}^{2}+x_{2}^{2}+y_{2}^{2}\right)^{2}} d x_{1} \wedge d y_{1}+2 \frac{\left(x_{1}^{2}+y_{1}^{2}\right)}{\left(x_{1}^{2}+y_{1}^{2}+x_{2}^{2}+y_{2}^{2}\right)^{2}} d x_{2} \wedge d y_{2} \\
&+2 \frac{x_{1} y_{2}-x_{2} y_{1}}{\left(x_{1}^{2}+y_{1}^{2}+x_{2}^{2}+y_{2}^{2}\right)^{2}} d x_{1} \wedge d x_{2}-2 \frac{x_{1} x_{2}+y_{1} y_{2}}{\left(x_{1}^{2}+y_{1}^{2}+x_{2}^{2}+y_{2}^{2}\right)^{2}} d x_{1} \wedge d y_{2} \\
&-2 \frac{x_{1} x_{2}+y_{1} y_{2}}{\left(x_{1}^{2}+y_{1}^{2}+x_{2}^{2}+y_{2}^{2}\right)^{2}} d x_{2} \wedge d y_{1}+2 \frac{x_{1} y_{2}-x_{2} y_{1}}{\left(x_{1}^{2}+y_{1}^{2}+x_{2}^{2}+y_{2}^{2}\right)^{2}} d y_{1} \wedge d y_{2} \\
& \text { i.e., } c_{11}=\frac{u_{2}}{\left(u_{1}+u_{2}\right)^{2}}, c_{22}=\frac{u_{1}}{\left(u_{1}+u_{2}\right)^{2}}, c_{12}=\frac{-u_{2}}{\left(u_{1}+u_{2}\right)^{2}}, c_{21}=\frac{-u_{1}}{\left(u_{1}+u_{2}\right)^{2}} \text { and } \\
& 1+c_{11}+c_{22}+c_{11} c_{22}-c_{12} c_{21}=1+\frac{u_{1}+u_{2}}{\left(u_{1}+u_{2}\right)^{2}} \neq 0 \text { in } \mathbf{R}^{4}-\{0\} .
\end{aligned}
$$


Consider $\Psi=\left(\Psi_{1}, \Psi_{2}\right)$ given by $\Psi_{1}=u_{1}+\frac{u_{1}}{u_{1}+u_{2}}, \Psi_{2}=u_{2}+\frac{u_{2}}{u_{1}+u_{2}}$. The perturbation $\mu=\Omega-\omega$ is homogeneous of degree zero, that is, $\left(\rho_{t}\right)^{*} \mu=$ $\mu$. Moreover, $\Psi \circ \rho_{t}\left(u_{1}, u_{2}\right)=\left(t u_{1}+\frac{u_{1}}{u_{1}+u_{2}}, t u_{2}+\frac{u_{2}}{u_{1}+u_{2}}\right)$ and $\lim _{t \rightarrow 0} \Psi \circ$ $\rho_{t}\left(u_{1}, u_{2}\right)=\left(\frac{u_{1}}{u_{1}+u_{2}}, \frac{u_{2}}{u_{1}+u_{2}}\right)=\left(f_{1}, f_{2}\right)$ where $\left(f_{1}, f_{2}\right)$ are points on the line $f_{1}+f_{2}=1$ with $f_{1}, f_{2} \geq 0$.

Hence, the image of $\Psi$ is the convex region in $\mathbf{R}^{2}$ shown in Figure 1 and the images of $\Phi$ and $\Psi$ are different.

Example 1.3. Let $X=\mathbf{R}^{4}-\{0\}$ and

$$
\begin{aligned}
& \Omega=\omega+2 \frac{\left(x_{2}^{2}+y_{2}^{2}\right)^{2}}{\left(x_{1}^{2}+y_{1}^{2}+x_{2}^{2}+y_{2}^{2}\right)^{\frac{3}{2}}} d x_{1} \wedge d y_{1}+2 \frac{\left(x_{1}^{2}+y_{1}^{2}\right)^{2}}{\left(x_{1}^{2}+y_{1}^{2}+x_{2}^{2}+y_{2}^{2}\right)^{\frac{3}{2}}} d x_{2} \wedge d y_{2} \\
& +\left(2 \frac{x_{1} y_{2}\left(x_{1}^{2}+y_{1}^{2}\right)}{\left(x_{1}^{2}+y_{1}^{2}+x_{2}^{2}+y_{2}^{2}\right)^{\frac{3}{2}}}-2 \frac{x_{2} y_{1}\left(x_{2}^{2}+y_{2}^{2}\right)}{\left(x_{1}^{2}+y_{1}^{2}+x_{2}^{2}+y_{2}^{2}\right)^{\frac{3}{2}}}\right) d x_{1} \wedge d x_{2} \\
& -\left(2 \frac{x_{1} x_{2}\left(x_{1}^{2}+y_{1}^{2}\right)}{\left(x_{1}^{2}+y_{1}^{2}+x_{2}^{2}+y_{2}^{2}\right)^{\frac{3}{2}}}+2 \frac{y_{1} y_{2}\left(x_{2}^{2}+y_{2}^{2}\right)}{\left(x_{1}^{2}+y_{1}^{2}+x_{2}^{2}+y_{2}^{2}\right)^{\frac{3}{2}}}\right) d x_{1} \wedge d y_{2} \\
& -\left(2 \frac{y_{1} y_{2}\left(x_{1}^{2}+y_{1}^{2}\right)}{\left(x_{1}^{2}+y_{1}^{2}+x_{2}^{2}+y_{2}^{2}\right)^{\frac{3}{2}}}+2 \frac{x_{1} x_{2}\left(x_{2}^{2}+y_{2}^{2}\right)}{\left(x_{1}^{2}+y_{1}^{2}+x_{2}^{2}+y_{2}^{2}\right)^{\frac{3}{2}}}\right) d x_{2} \wedge d y_{1} \\
& +\left(-2 \frac{x_{2} y_{1}\left(x_{1}^{2}+y_{1}^{2}\right)}{\left(x_{1}^{2}+y_{1}^{2}+x_{2}^{2}+y_{2}^{2}\right)^{\frac{3}{2}}}+2 \frac{x_{1} y_{2}\left(x_{2}^{2}+y_{2}^{2}\right)}{\left(x_{1}^{2}+y_{1}^{2}+x_{2}^{2}+y_{2}^{2}\right)^{\frac{3}{2}}}\right) d y_{1} \wedge d y_{2}
\end{aligned}
$$

i.e. $\quad c_{11}=\frac{u_{2}^{2}}{\left(u_{1}^{2}+u_{2}^{2}\right)^{\frac{3}{2}}}, c_{22}=\frac{u_{1}^{2}}{\left(u_{1}^{2}+u_{2}^{2}\right)^{\frac{3}{2}}}, c_{12}=c_{21}=-\frac{u_{1} u_{2}}{\left(u_{1}^{2}+u_{2}^{2}\right)^{\frac{3}{2}}}$. The form $\Omega$ is non-degenerate since $1+c_{11}+c_{22}+c_{11} c_{22}-c_{12} c_{21}=1+\frac{u_{1}^{2}+u_{2}^{2}}{\left(u_{1}^{2}+u_{2}^{2}\right)^{\frac{3}{2}}} \neq 0$ in $\mathbf{R}^{4}-\{0\}$

Let $\Psi=\left(\Psi_{1}, \Psi_{2}\right)$ where $\Psi_{1}=u_{1}+\frac{u_{1}}{\sqrt{u_{1}^{2}+u_{2}^{2}}}$ and $\Psi_{2}=u_{2}+\frac{u_{2}}{\sqrt{u_{1}^{2}+u_{2}^{2}}}$.

The perturbation $\mu=\Omega-\omega$ is such that $\left(\rho_{t}\right)^{*} \mu=\mu$ and the moment map $\Psi$ satisfies $\Psi \circ \rho_{t}\left(u_{1}, u_{2}\right)=\left(t u_{1}+\frac{u_{1}}{\sqrt{u_{1}^{2}+u_{2}^{2}}}, t u_{2}+\frac{u_{2}}{\sqrt{u_{1}^{2}+u_{2}^{2}}}\right)$. Therefore, $\lim _{t \rightarrow 0} \Psi \circ \rho_{t}\left(u_{1}, u_{2}\right)=\left(\frac{u_{1}}{\sqrt{u_{1}^{2}+u_{2}^{2}}}, \frac{u_{2}}{\sqrt{u_{1}^{2}+u_{2}^{2}}}\right)=\left(g_{1}, g_{2}\right)$ where $\left(g_{1}, g_{2}\right)$ are points on the circle $g_{1}^{2}+g_{2}^{2}=1$ with $g_{1}, g_{2} \geq 0$.

It follows then that the image of $\Psi$ is the non-convex region in $\mathbf{R}^{2}$ indicated in Figure 2 and the images of $\Psi$ and $\Phi$ are different. 


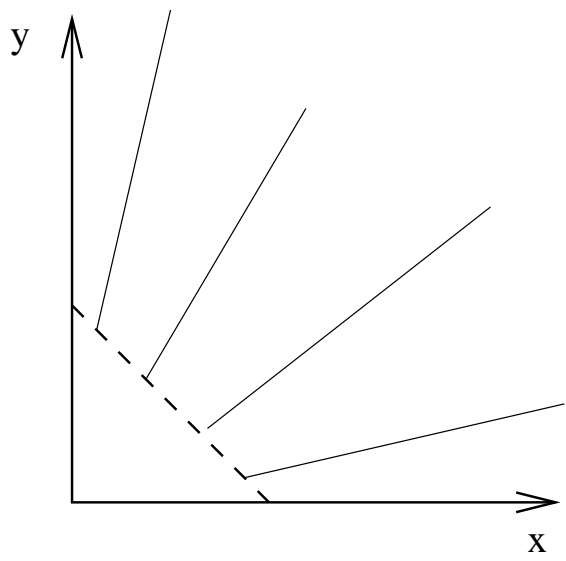

Figure 1.

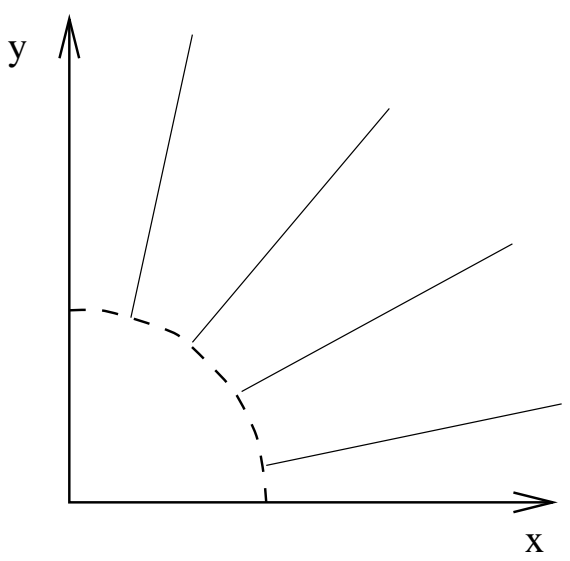

Figure 2.

In the next example, the images of the moment maps close to infinity are different polytopic cones. This is a consequence of the behaviour of the perturbation close to the origin.

Example 2. Consider $\mathbf{C}^{4}-\{0\}$ with symplectic form

$$
\omega=\frac{\sqrt{-1}}{2}\left(d z_{1} \wedge d \overline{z_{1}}+d z_{2} \wedge d \overline{z_{2}}-d z_{3} \wedge d \overline{z_{3}}-d z_{4} \wedge d \overline{z_{4}}\right)
$$

The Hamiltonian action of $S^{1}$ on $\left(\mathbf{C}^{4}-\{0\}, \omega\right)$ given by

$$
e^{i \theta} \cdot\left(z_{1}, z_{2}, z_{3}, z_{3}, z_{4}\right)=\left(e^{i \theta} \cdot z_{1}, e^{i \theta} \cdot z_{2}, e^{i \theta} \cdot z_{3}, e^{i \theta} \cdot z_{4}\right)
$$

admits $H\left(z_{1}, z_{2}, z_{3}, z_{4}\right)=\frac{1}{2}\left(\left|z_{1}\right|^{2}+\left|z_{2}\right|^{2}-\left|z_{3}\right|^{2}-\left|z_{4}\right|^{2}\right)$ as a moment map and $H^{-1}(0)=\left\{\left(z_{1}, z_{2}, z_{3}, z_{4}\right) \in \mathbf{C}^{4}-\left.\{0\}|| z_{1}\right|^{2}+\left|z_{2}\right|^{2}=\left|z_{3}\right|^{2}+\left|z_{4}\right|^{2}\right\}$ is a manifold.

Let $\rho_{t}\left(z_{1}, z_{2}, z_{3}, z_{4}\right)=\left(\sqrt{t} \cdot z_{1}, \sqrt{t} \cdot z_{2}, \sqrt{t} \cdot z_{3}, \sqrt{t} \cdot z_{4}\right)$ for $t \in \mathbf{R}^{+}$be an action of $\mathbf{R}^{+}$on $\mathbf{C}^{4}-\{0\}$.

It is easy to see that $\mathbf{R}^{+}$and $S_{1}$ act on $H^{-1}(0)$, that these actions commute and that $\left(\rho_{t}\right)^{*} \omega=t \cdot \omega$. Therefore, if $X$ is the reduced space $H^{-1}(0) / S^{1}, \mathbf{R}^{+}$ also acts on $X$.

If $\omega_{0}$ is the symplectic form on $X$ induced by $\omega$, then $\left(\rho_{t}\right)^{*} \omega_{0}=t \cdot \omega_{0}$ and $X / \mathbf{R}^{+} \simeq H^{-1}(0) / \mathbf{C}^{*}$ is a compact manifold. Hence, $\left(X, \omega_{0}\right)$ is a symplectic cone with base $H^{-1}(0) / \mathbf{C}^{*}$.

Consider the Hamiltonian action of the torus $T^{3}$ on $\left(X, \omega_{0}\right)$ given by

$$
\left(e^{i \theta_{1}}, e^{i \theta_{2}}, e^{i \theta_{3}}\right) \cdot\left[z_{1}, z_{2}, z_{3}, z_{4}\right]=\left[e^{i \theta_{1}} \cdot z_{1}, e^{i \theta_{2}} \cdot z_{2}, e^{i \theta_{3}} \cdot z_{3}, z_{4}\right]
$$

where $\left(z_{1}, z_{2}, z_{3}, z_{4}\right)$ is a point in $H^{-1}(0)$ and $\left[z_{1}, z_{2}, z_{3}, z_{4}\right]$ is the corresponding point in $X$. 
Let $\Phi: X \rightarrow \mathbf{R}^{3}$ be the associated moment map (normalized in zero). Then

$$
\Phi\left(\left[z_{1}, z_{2}, z_{3}, z_{4}\right]\right)=\left(\frac{\left|z_{1}\right|^{2}}{2}, \frac{\left|z_{2}\right|^{2}}{2},-\frac{\left|z_{3}\right|^{2}}{2}\right)
$$

with image

$$
\operatorname{Im} \Phi=\left\{(x, y,-z) \in \mathbf{R}^{3}-\{0\} \mid x+y \geq z, x, y, z \geq 0\right\}
$$

a polytopic convex cone with vertex at the origin, bounded by the planes $x=0, y=0, x+y=-z$. Notice that the intersection of $\operatorname{Im}(\Phi)$ with the family of planes $x+y=c$ ( $c$ real and greater than zero) is a family of rectangles with sides $c$ and $c \sqrt{2}$.

Define now in $\mathbf{C}^{4}-\left\{\left(z_{1}, z_{2}, z_{3}, z_{4}\right)\right.$ such that $z_{1}=z_{2}=0$ or $\left.z_{3}=z_{4}=0\right\}$ the symplectic form

$$
\begin{aligned}
\Omega=\omega+\frac{\sqrt{-1}}{2}( & \frac{\left|z_{2}\right|^{2}}{\left(\left|z_{1}\right|^{2}+\left|z_{2}\right|^{2}\right)^{2}} d z_{1} \wedge d \overline{z_{1}}+\frac{\left|z_{1}\right|^{2}}{\left(\left|z_{1}\right|^{2}+\left|z_{2}\right|^{2}\right)^{2}} d z_{2} \wedge d \overline{z_{2}} \\
& -\frac{2\left|z_{4}\right|^{2}}{\left(\left|z_{3}\right|^{2}+\left|z_{4}\right|^{2}\right)^{2}} d z_{3} \wedge d \overline{z_{3}}-\frac{2\left|z_{3}\right|^{2}}{\left(\left|z_{3}\right|^{2}+\left|z_{4}\right|^{2}\right)^{2}} d z_{4} \wedge d \overline{z_{4}} \\
& -\frac{z_{1} z_{2}}{\left(\left|z_{1}\right|^{2}+\left|z_{2}\right|^{2}\right)^{2}} d z_{1} \wedge d \overline{z_{2}}-\frac{z_{1} \overline{z_{2}}}{\left(\left|z_{1}\right|^{2}+\left|z_{2}\right|^{2}\right)^{2}} d z_{2} \wedge d \overline{z_{1}} \\
& \left.+\frac{2 \overline{z_{3}} z_{4}}{\left(\left|z_{3}\right|^{2}+\left|z_{4}\right|^{2}\right)^{2}} d z_{3} \wedge d \overline{z_{4}}+\frac{2 z_{3} \overline{z_{4}}}{\left(\left|z_{3}\right|^{2}+\left|z_{4}\right|^{2}\right)^{2}} d z_{4} \wedge d \overline{z_{3}}\right) .
\end{aligned}
$$

Let $\Omega_{0}$ be the symplectic form induced by $\Omega$ in $X$. It is easy to see that the action of $T^{3}$ on $\left(X, \Omega_{0}\right)$ is still Hamiltonian with associated moment map $\Psi: X \rightarrow \mathbf{R}^{3}$ where

$$
\begin{aligned}
& \Psi\left(\left[z_{1}, z_{2}, z_{3}, z_{4}\right]\right) \\
& =\frac{1}{2}\left(\left|z_{1}\right|^{2}+\frac{\left|z_{1}\right|^{2}}{\left|z_{1}\right|^{2}+\left|z_{2}\right|^{2}},\left|z_{2}\right|^{2}+\frac{\left|z_{2}\right|^{2}}{\left|z_{1}\right|^{2}+\left|z_{2}\right|^{2}},-\left|z_{3}\right|^{2}-\frac{2\left|z_{3}\right|^{2}}{\left|z_{3}\right|^{2}+\left|z_{4}\right|^{2}}\right)
\end{aligned}
$$

with image

$$
\operatorname{Im} \Psi=\left\{(x, y,-z) \in \mathbf{R}^{3} \mid x, y, z \geq 0, x+y>1, x+y+1 \geq z\right\}
$$

a truncated cone (still polytopic and convex) in $\mathbf{R}^{3}$ bounded by the planes $x=0, y=0, x+y=z+1, x+y=1$. The intersection of $\operatorname{Im}(\Psi)$ with the family of planes $x+y=c$ ( $c$ real and greater than 1$)$ is a family of rectangles with sides $c \sqrt{2}$ and $c+1$.

In this example, the two images are different and it is not possible to include by translation one into the other. The images are shown in Figure 3 . 


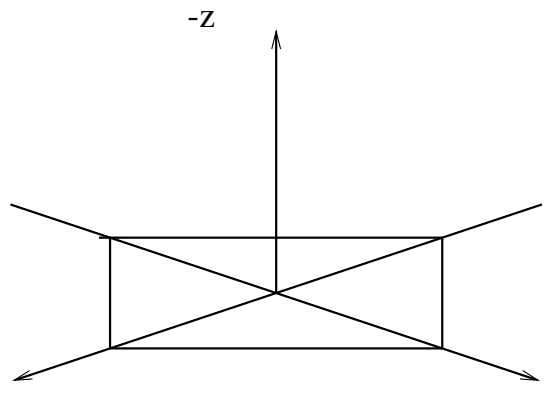

the original image at infinity

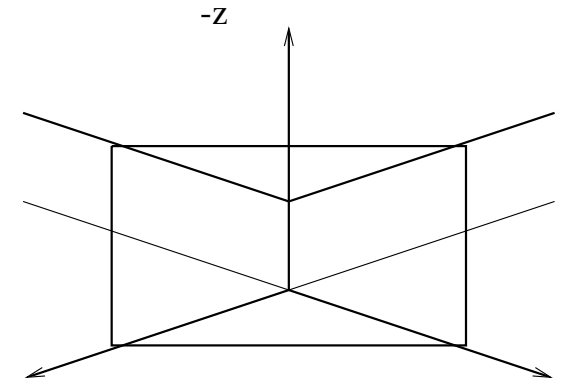

the perturbed image at infinity

Figure 3.

\section{References}

[1] R. Abraham and J. Marsden, Foundations of mechanics, Benjamin, 1978, Reading, MA.

[2] M.F. Atiyah, Convexity and commuting Hamiltonians, Bull. London Math. Soc., 14 (1982), 1-15.

[3] R. Bott, Nondegenerate critical manifolds, Ann. Math., 60(2) (1959), 248-261.

[4] V. Guillemin and S. Sternberg, Convexity properties of the moment mapping, Invent. Math., 67 (1982), 491-513.

[5] A Normal form for the moment map, Diff. Geom. Methods, (ed. S.Sternberg), Mathematical Physical Studies, Vol. 6, (1984), 161-175, D. Reidel, Boston.

[6] Symplectic techniques in physics, (1984), Cambridge University Press, Cambridge.

[7] E. Lerman, On the centralizer of invariant functions on a Hamiltonian G-space, J. of Diff. Geom., 30 (1989), 805-815.

[8] C.M. Marle, Classification des actions hamiltoniennes au voisinage d'une orbite, C. R. Acad. Sci. Paris, Ser. I, Math., 299 (1984) 249-252.

[9] G.D. Mostow, On a conjecture of Montgomery, Ann. of Math., 65(2) (1957), 513516.

[10] E. Prato, Convexity properties of the moment map for certain non - compact manifolds, preprint.

Received May 20, 1993 and revised November 17, 1996.

PUC-RIO

R. Marquês DE S. Vicente, 225

Rio DE JANEIRO, 22453-900

BRAZIL

E-mail address: tomei@mat.puc-rio.br 